\title{
An Effelsberg HI study of the ISM around WR 126, WR 154 and WR 155^
}

\author{
S. Cichowolski ${ }^{1}$ and E. M. Arnal ${ }^{1,2}$ \\ 1 Instituto Argentino de Radioastronomía, CC \# 5, 1894 Villa Elisa, Argentina \\ e-mail: silvina@iar.unlp.edu.ar ${ }^{\star \star}$ \\ 2 Facultad de Ciencias Astronómicas y Geofísicas, Universidad Nacional La Plata, 1900 La Plata, Argentina \\ e-mail: arnal@iar.unlp.edu. ar ${ }^{\star \star \star}$
}

Received 5 May 2003 / Accepted 22 August 2003

\begin{abstract}
The neutral hydrogen distribution has been studied in the direction of three Galactic Wolf-Rayet (WR) stars using the $100 \mathrm{~m}$ Effelsberg radio telescope. Cavities in the HI distribution, regions of low HI emissivity, are observed over a 8-9 $\mathrm{km} \mathrm{s}^{-1}$ velocity range for WR 126 (三ST 2), WR 154 (三HD 213049) and WR 155 (三HD 214419). These minima are interpreted as the observable $21-\mathrm{cm}$ HI line counterpart of interstellar bubbles created by the winds of the WR stars and their progenitors. The HI cavities are elongated structures depicting an axial ratio ranging from 1.3 (WR 155) to 3 (WR 126). The WR stars are always eccentric with respect to either the geometric centre of the HI cavity or the absolute minimum inside it. This offset ranges from $50 \%$ to $80 \%$ of the HI hole's minor axis. The major axis of these structures range from 13 (WR 155) to $27 \mathrm{pc}$ (WR 126), while the missing HI mass amounts to 45-50 $M_{\odot}$ (WR 126), $60 M_{\odot}$ (WR 155) and $85 M_{\odot}$ (WR 154).
\end{abstract}

Key words. ISM: bubbles - stars: Wolf-Rayet - stars: individual: WR 126, WR 154, WR 155

\section{Introduction}

This is the fifth in a series of papers dealing with intermediate $\left(\right.$ HPBW $\sim 9^{\prime}$ ) angular resolution of $\lambda \sim 21 \mathrm{~cm}$ line observations towards galactic Wolf-Rayet (WR) stars. Previous papers of this series (Arnal 1992; Cappa et al. 1996; Arnal \& Cappa 1996; Arnal et al. 1999) have provided direct evidence for the existence of ovoidal HI minima in the interstellar medium (ISM) local to the surveyed stars. It is believed that these HI minima are an observational manifestation of interstellar bubbles (IB) created by those stars. In those HI minima related to WR 6 (Arnal \& Cappa 1996), WR 3 (Arnal \& Roger 1997) and WR 140 (Arnal 2001), evidence suggesting the existence of small scale structure within the main minimum has been provided.

It is well known that over their lifetimes, massive stars have a profound influence on their local ISM by heating it, changing its chemical composition and disturbing its dynamics. This major impact is due to three circumstances, namely, $i$ ) their high mass loss rates, ii) their strong ultraviolet radiation field; and iii) by ending their life with a huge injection of energy into their local ISM: the final disruption of the star by a supernova explosion.

Wolf-Rayet stars, the last evolutionary phase of stars having masses above $22 M_{\odot}$ for $\mathrm{Z}=0.02$ (Meynet \& Maeder 2003),

* Figures 1, 3, 5 are only available in electronic form at http://www.edpsciences.org

$\star \star$ Fellowship from CONICET, Argentina.

$\star \star \star$ Member of Carrera del Investigador Científico, CONICET, Argentina. have lost a significant fraction of their outer envelopes via their strong stellar winds. Optical, infrared and radio studies have shown that these stars have powerful winds with mass-loss rates of (3-5) $\times 10^{-5} M_{\odot} \mathrm{yr}^{-1}$ and wind velocities in the range from 1000 to $3000 \mathrm{~km} \mathrm{~s}^{-1}$ (van der Hucht 2001).

Based on the above numbers, the mechanical energy injected by the winds of massive stars over their lifetimes is of the order of a few times $10^{51} \mathrm{ergs}$, which is comparable to the energy released by a single supernova explosion.

The first complete theoretical study of the interaction between a stellar wind and the surrounding interstellar gas was done by Avedisova (1972), followed by Castor et al. (1975) and Weaver et al. (1977). They predict the creation around a high mass-loss rate star of a huge region having a very low volume density $\left(10^{-2}-10^{-3} \mathrm{~cm}^{-3}\right)$ and a high temperature $\left(10^{6}-10^{7} \mathrm{~K}\right)$. This structure is known as an interstellar bubble (IB). Analytical models for the evolution of IB have been developed under a variety of interstellar environments and evolutive stages of the central star (García-Segura \& Mac Low 1995a, 1995b and references therein).

In this paper we discuss observations of the $\lambda \sim 21 \mathrm{~cm}$ HI line towards the galactic WR stars WR 126, WR 154 and WR 155 (van der Hucht 2001), using the $100 \mathrm{~m}$ Effelsberg radiotelescope of the Max-Planck Institut für Radioastronomie in Bonn, Germany.

\section{Observations}

The HI data were collected in August 1991. For each WR star, a $2^{\circ} \times 2^{\circ}$ region centred at the stellar optical position was 
Table 1. Stellar parameters.

\begin{tabular}{lcccc}
\hline \hline Name & $l$ & $b$ & Sp. Class $^{b}$ & $\mathrm{~d}(\mathrm{kpc})$ \\
\hline WR 126 & 61.89 & +2.11 & WC5/WN & $4.4^{a}$ \\
& & & & $4.41^{b}$ \\
& & & & $3.78^{c}$ \\
& & & & $2.31^{c}$ \\
WR 154 & 103.85 & -1.18 & WC6 & $4.3^{a}$ \\
& & & & $2.75^{b}$ \\
& & & & $3.21^{c}$ \\
WR 155 & \multirow{2}{*}{$105^{\circ} .32$} & -1.29 & WN6 + O9II-Ib & $3.8^{a}$ \\
& & & & $2.75^{b}$
\end{tabular}

${ }^{a}$ Conti \& Vacca (1990).

${ }^{b}$ van der Hucht (2001).

${ }^{c}$ Smith et al. (1990).

observed. Within each region, the observed points were sampled at different spacings. An inner grid $1^{\circ} \times 1^{\circ}$ in size has a grid spacing of $5^{\prime}$ while outside it the spacing is $10^{\prime}$. The HPBW and main-beam efficiency at $\lambda \sim 21 \mathrm{~cm}$ are $9^{\prime}$ and 0.72 , respectively. During the observing run the dual-channel front end consisted of field effect transistor amplifiers, yielding a system temperature against cold sky of about $30 \mathrm{~K}$. A 1024-channel autocorrelator, split into two receivers with 512 channels each, was used as back-end. The frequency-switching technique with the reference band set $1.6 \mathrm{MHz}$ above the signal band was used. This configuration yields a velocity coverage of $\sim 330 \mathrm{~km} \mathrm{~s}^{-1}$ and a velocity resolution of $0.77 \mathrm{~km} \mathrm{~s}^{-1}$. The integration time per point was $16 \mathrm{~s}$. The rms noise level varies between 0.25 and $0.35 \mathrm{~K}$ in main-beam brightness temperature units. This scale was derived from observations of the IAU standard region S7 $\left[(l, b)=\left(132^{\circ} .0,-1^{\circ} 0\right)\right]$ (Williams 1973). All profiles were corrected for stray radiation by applying the procedure outlined by Kalberla et al. (1980). The overall brightness temperature scale is accurate to $2 \%-3 \%$.

\section{Analysis of the observations}

\subsection{Searching for the bubbles}

The main data for the stars are summarized in Table 1. The first column lists the name of the star; Cols. 2 and 3, its galactic coordinates; Col. 4, the spectral classification as given by van der Hucht (2001), and Col. 5, the stellar distance as given by different authors.

As was described in previous papers of this series, in order to analyse the gas distribution and dynamics in the ISM local to our target WR stars, a series of $(l, b)$ images, showing the $\mathrm{HI}$ emission distribution at different velocities were constructed for each field. For the sake of presentation, a constant background corresponding to the average brightness temperature within the entire image was subtracted from every individual line channel map in every data cube. These images were carefully inspected for the existence of either HI shells or cavities that might be related to the stars. Three basic requirements have to be fulfilled by a given minimum in the observed HI distribution to stand the chance of being physically related to the WR star, namely: $i$ ) the HI structure should remain detectable along a velocity interval larger than the ISM turbulent velocity $\left.\left(\sim 8 \mathrm{~km} \mathrm{~s}^{-1}\right), i i\right)$ the WR star should be seen projected onto, or close to, the centre of the HI minimum; and iii) the kinematical distance derived for the HI structure should be compatible, within errors, with one of the catalogued distances of the star. Bearing in mind that the existence of peculiar motions in the interstellar gas may result in an erroneous kinematical distance assignment, and that different distance estimates for a given WR star agree to within a factor of $\sim 2$ (e.g., van der Hucht 2001; Conti \& Vacca 1990; Smith et al. 1990), lower weight is usually given to the last criterion.

\subsection{Properties of the bubbles}

In order to characterize every HI minimum possibly related to a given WR star, many parameters are derived. Firstly, the size of the cavity can be expressed by its major $\left(d_{\mathrm{M}}\right)$ and minor $\left(d_{\mathrm{m}}\right)$ axis. Then, two parameters are defined: the ratio of the major to minor axis, $C_{\mathrm{ax}}=d_{\mathrm{M}} / d_{\mathrm{m}}$, and the offset parameter, $C_{\text {off }}$, which is the ratio of the observed distance between the WR star and the symmetry center (SC) of the HI minimum (also referred to as $\mathrm{HI}$ cavity) to its minor axis $\left(d_{\mathrm{m}}\right)$. By placing the minor axis midway along the major axis, we use the crossing point to define the SC. Owing to projection effects, both $C_{\mathrm{ax}}$ and $C_{\text {off }}$ may be lower limits.

To define the angular extent of the HI cavity, we adopt the first nonclosed contour line defining the void. Under the assumption that the HI gas is optically thin, the missing HI mass $\left(M_{\mathrm{HI}}^{\mathrm{miss}}\right)$ of a feature located at a distance of $d \mathrm{kpc}$ covering a solid angle of $\Omega$ square arc minutes, is given by (Pineault 1998)

$M_{\mathrm{HI}}^{\mathrm{miss}} \simeq 1.3 \times 10^{-3} d^{2} \Delta v \overline{\Delta T_{\mathrm{B}}} \Omega \quad\left(M_{\odot}\right)$

where $\Delta v$ is the velocity interval, in units of $\mathrm{km} \mathrm{s}^{-1}$, over which the HI cavity is visible. The mean brightness temperature $\overline{\Delta T_{\mathrm{B}}}$ is defined as $\overline{\Delta T_{\mathrm{B}}}=\left|T_{\text {cav }}-T_{\mathrm{bg}}\right|$, where $T_{\text {cav }}$ refers to the mean brightness temperature of the $\mathrm{HI}$ cavity, and $T_{\mathrm{bg}}$ corresponds to the temperature of the contour level defining the outer border of the HI minimum and represents the temperature of the neighbouring gas. The interval $\Delta v$ (and therefore $M_{\mathrm{HI}}^{\text {miss }}$ as well) is a lower limit to the real velocity extent of the HI void, because "extreme"-velocity gas associated with the cavity could be missed due to both confusing effects and the intrinsic weakness of the HI emission at those velocities. The missing HI density is obtained by distributing the missing $\mathrm{HI}$ mass, $M_{\mathrm{HI}}^{\text {miss }}$, all over the volume of the cavity. Assuming this mass to be distributed in an ovoidal volume whose major axis is $d_{\mathrm{M}}$ and the other two dimensions are set equal to $d_{\mathrm{m}}$, the mean missing volume density is obtained from (Arnal et al. 1999)

$n_{\mathrm{HI}}^{\mathrm{miss}} \simeq 79.2 \frac{M_{\mathrm{HI}}^{\mathrm{miss}}}{d_{\mathrm{M}} d_{\mathrm{m}}^{2}} \quad\left(\mathrm{~cm}^{-3}\right)$

where $M_{\mathrm{HI}}^{\mathrm{miss}}$ is given in $M_{\odot}$, and $d_{\mathrm{M}}$ and $d_{\mathrm{m}}$ in pc. The systemic velocity, $V_{\text {sys }}$, represents the central value of the velocity range $(\Delta v)$ covered by the $\mathrm{HI}$ depression. Using 
Table 2. Parameters of the HI structures related to the WR stars.

\begin{tabular}{lcccc}
\hline \hline Parameter & \multicolumn{2}{c}{ WR 126 } & WR 154 & WR155 \\
& Cavity 2 & Dual-lobe & & \\
\hline Longitude of the SC $(\mathrm{deg})$ & 61.9 & 61.9 & 103.7 & 105.4 \\
Latitude of the SC $(\mathrm{deg})$ & 2.2 & 2.25 & -1.3 & -1.2 \\
Systemic velocity $V_{\text {sys }}\left(\mathrm{km} \mathrm{s}^{-1}\right)$ & 23 & 23 & -29 & -29 \\
Velocity extent $\left(\mathrm{km} \mathrm{s}^{-1}\right)$ & 8 & 8 & 9 & 9 \\
Kinematical distance $\left(d_{\mathrm{k}}\right)(\mathrm{kpc})$ & 2.6 & 2.6 & 2.0 & 2.0 \\
Major axis $\left(d_{\mathrm{M}}\right)(\mathrm{pc})$ & 18 & 27 & 15 & 13 \\
Minor axis $\left(d_{\mathrm{m}}\right)(\mathrm{pc})$ & 9 & 9 & 10 & 10 \\
Axial ratio $\left(C_{\mathrm{ax}}\right)$ & 2 & 3 & 1.5 & 1.3 \\
Offset parameter $\left(C_{\text {off }}\right)$ & 0.5 & 0.8 & 0.7 & 0.5 \\
Missing HI mass $\left(M_{\mathrm{HI}}^{\text {miss }}\right)\left(M_{\odot}\right)$ & 45 & 50 & 85 & 60 \\
Missing HI density $\left(n_{\mathrm{HI}}^{\text {miss }}\right)\left(\mathrm{cm}^{-3}\right)$ & 2.5 & 2.0 & 4.5 & 4.0 \\
\hline
\end{tabular}

$V_{\text {sys }}$ in conjunction with different rotation galactic models (e.g. Ostriker \& Caldwell 1983; Blitz 1979), a kinematical distance $d_{\mathrm{k}}$ is obtained. To take into account the possible presence of non circular motions, the observed radial velocity field of the Galaxy derived by Brand \& Blitz (1993) was used as anoher way to estimate the kinematical distance of a given HI structure. Since the later is derived from an observed velocity field, higher weight is given to it. The quoted uncertainties in $d_{\mathrm{k}}$ are derived assuming turbulent motions of the order of $\pm 8 \mathrm{~km} \mathrm{~s}^{-1}$. Neutral hydrogen masses, densities and linear dimensions are evaluated using $d_{\mathrm{k}}$. Overall results are listed in Table 2 .

\section{Results and discussion}

\subsection{The ISM local to WR 126}

WR 126 (ST2) is a star classified as a WC5/WN and it is listed as a probable member of the VulOB2 association (van der Hucht 2001).

In their $\mathrm{H} \alpha$ and [OIII] $\lambda 5007 \AA$ survey no optical nebulosity likely to be related to this star was detected by Miller \& Chu (1993). An inspection of the IRAS images at $60 \mu \mathrm{m}$ and $100 \mu \mathrm{m}$ (Wheelock et al. 1994) also fails to show significant emission in its environment.

A thorough search throughout the available radio continuum surveys unveils the presence of two point-like radio sources, $1936+2639$ and $1937+2640$, located $17 ! 2$ and $14 ! 2$ away from the optical position of WR 126, respectively. Besides these sources, no extended continuum emission is present in this region. The spectral index of $1936+2639$ is steeper than -0.9 (White \& Becker 1992). As for $1937+2640$, it appears as a point source at $4850 \mathrm{MHz}$ with a total flux density of $65 \mathrm{mJy}$ (Condon et al. 1989), whilst in the NRAO VLA Sky Survey at $1420 \mathrm{MHz}$ it is resolved out in two sources with individual flux densities of $65.4 \pm 2.4$ and $108.2 \pm 4.2 \mathrm{mJy}$. Using the integrated flux density at $1420 \mathrm{MHz}$, a composite spectral index of -0.8 is derived for this source. It is important to note that if one of the two sources observed at $1420 \mathrm{MHz}$ were thermal in nature, the flux density that should be observable at $4850 \mathrm{MHz}$ would be much higher. Since both sources have a steep spectral index, very likely both represent extragalactic objects, that happen to be projected close to WR 126. Based on this, hereafter we shall not consider them any further.

\subsubsection{Analysis of the $\mathrm{HI}$ data}

After inspecting the entire HI data cube, from -85 to $40 \mathrm{~km} \mathrm{~s}^{-1}$, we ended up focusing our attention on the velocity range from 10 to $30 \mathrm{~km} \mathrm{~s}^{-1}$. In Fig. 1 a series of images showing the $\mathrm{HI}$ brightness temperature distribution along the velocity range 14.9 to $25.2 \mathrm{~km} \mathrm{~s}^{-1}$ is shown.

At $16.2 \mathrm{~km} \mathrm{~s}^{-1}$, a well developed HI minimum centred at $(l, b)=\left(62^{\circ} .0,2.3\right)$ is easily recognizable. This minimum is surrounded by features of enhanced HI emissivity and the WR star is observed projected close to, though interior, the regions of high HI emissivity that define the outer boundary of the HI minimum. We shall refer to this structure as Cavity 1. From the same set of images, one may get the impression that another minimum centred at $(l, b)=\left(62^{\circ} .2,2^{\circ} .0\right)$ is closely related to Cavity 1 . Nevertheless, this minimum is first detected at $7 \mathrm{~km} \mathrm{~s}^{-1}$ and persist over $13 \mathrm{~km} \mathrm{~s}^{-1}$. Based on this, we believe that a physical relationship between this feature and Cavity 1 appears as highly unlikely.

It is worth mentioning that Cavity 1 weakens as we move towards positive velocities, it loses its identity as a separate feature around $21 \pm 2 \mathrm{~km} \mathrm{~s}^{-1}$ and becomes again a well defined structure from $23 \mathrm{~km} \mathrm{~s}^{-1}$ till $26 \mathrm{~km} \mathrm{~s}^{-1}$ where it disappears as a recognizable structure.

At $20 \mathrm{~km} \mathrm{~s}^{-1}$, another $\mathrm{HI}$ minimum sets in centred at $(l$, $b)=\left(61^{\circ} .9,2.2\right)$. This feature is best defined around $23 \mathrm{~km} \mathrm{~s}^{-1}$ and remains visible till $25.2 \mathrm{~km} \mathrm{~s}^{-1}$. The WR star has a very good positional agreement with this HI minimum that, from here onwards, will be referred to as Cavity 2.

At $l \sim 62^{\circ}$ and taking into account the catalogued distances of the star (see Table 1), different galactic rotation models (e.g. Ostriker \& Caldwell 1983; Blitz 1979) predict a 
radial velocity of $21 \pm 3 \mathrm{~km} \mathrm{~s}^{-1}$ for a distance of $2.3 \mathrm{kpc}$ and about $29 \pm 4 \mathrm{~km} \mathrm{~s}^{-1}$ for a distance of $4.4 \mathrm{kpc}$. Thus, the systemic radial velocity adopted for Cavity $2, V_{\text {sys }}=23 \mathrm{~km} \mathrm{~s}^{-1}$, is in relative good agreement with both distance estimates. On the other hand, an inspection of the observed radial velocity field of the Galaxy (see Fig. 2b of Brand \& Blitz 1993) along $l \sim 62^{\circ}$, clearly shows the presence of large scale non-circular motions. From this velocity field, we can infer that along $l \sim$ $62^{\circ}$, for $v \sim 23 \mathrm{~km} \mathrm{~s}^{-1}$ the close and far kinematical distances are $\sim 1.3$ and $\sim 2.6 \mathrm{kpc}$, respectively. Given the apparent importance of non-circular motions along $l \sim 62^{\circ}$, and the agreement between the far kinematical distance, $2.6 \mathrm{kpc}$, and the $2.3 \mathrm{kpc}$ distance estimate quoted by Smith et al. (1990), we shall adopt for Cavity 2 a distance of $2.6 \mathrm{kpc}$. This distance is also compatible with the absolute magnitude expected for a WC5/WN star (van der Hucht 2001). Indeed, assuming for WR 126 a visual magnitude of 13.29 and a visual absorption of 3.9 (van der Hucht 2001), an absolute magnitude of -2 . 7 is obtained when a distance of $2.6 \mathrm{kpc}$ is considered. Since the distance of Vul OB2 is about $4.1 \mathrm{kpc}$ (Ruprecht et al. 1981), this would imply that WR 126 is not a member of this association.

It is important to mention that there are no catalogued early type stars in the vicinity of WR126 capable of having contributed to the creation of the observed HI structure.

Based on these findings, we believe that Cavity 2 may be regarded as the HI fingerprint of the interestellar bubble associated with WR 126. On the other hand, the velocity behaviour of Cavity 1 is quite puzzling. Although at $20 \mathrm{~km} \mathrm{~s}^{-1}$ it is hardly detectable, it regains strength at the same velocity where Cavity 2 begins to be observable. This fact could be understood within a scenario where Cavity 1 may be interpreted as a pre-existing structure that is being re-energized by the action of the stellar wind of WR 126 through the interaction with Cavity 2. This explanation would easily account for the dual lobed structure seen in Fig. 2, where the mean brightness temperature image in the velocity range from 20.1 to $25.2 \mathrm{~km} \mathrm{~s}^{-1}$ is shown. Since the above interpretation is highly speculative, for the sake of completeness in Table 2 we shall give separately the physical parameteres of both Cavity 2 and the HI dual-lobed feature.

\subsection{The ISM surrounding WR 154 and WR155}

WR 154 (HD 213049) and WR 155 (CQ Cep, HD 214419) are WC 6 and WN 6+O9 II-Ib stars, respectively (van der Hucht 2001). Together with WR 152 and WR 153, they are consider as probable members of Cep OB1 (Lundström \& Stenholm 1984). WR 152 and WR 153 may be associated with the HII region Sh 132 (Harten et al. 1978). Garmany \& Stencel (1992) derived for Cep OB1 a distance of $2.75 \mathrm{kpc}$.

Since four WR stars may be located at a similar distance, due to the cumulative effect of their stellar winds there is a chance that a structure larger than the $2^{\circ} \times 2^{\circ}$ field observed around every individual WR star could have been created by their collective action. In order to possibly identify such a structure, we have inspected the HI survey of Weaver \& Williams (1973) to analyze the HI distribution in the region $l=96^{\circ}$ to $109^{\circ}$ and $b=-6^{\circ}$ to $3^{\circ}$. This survey is well suited for

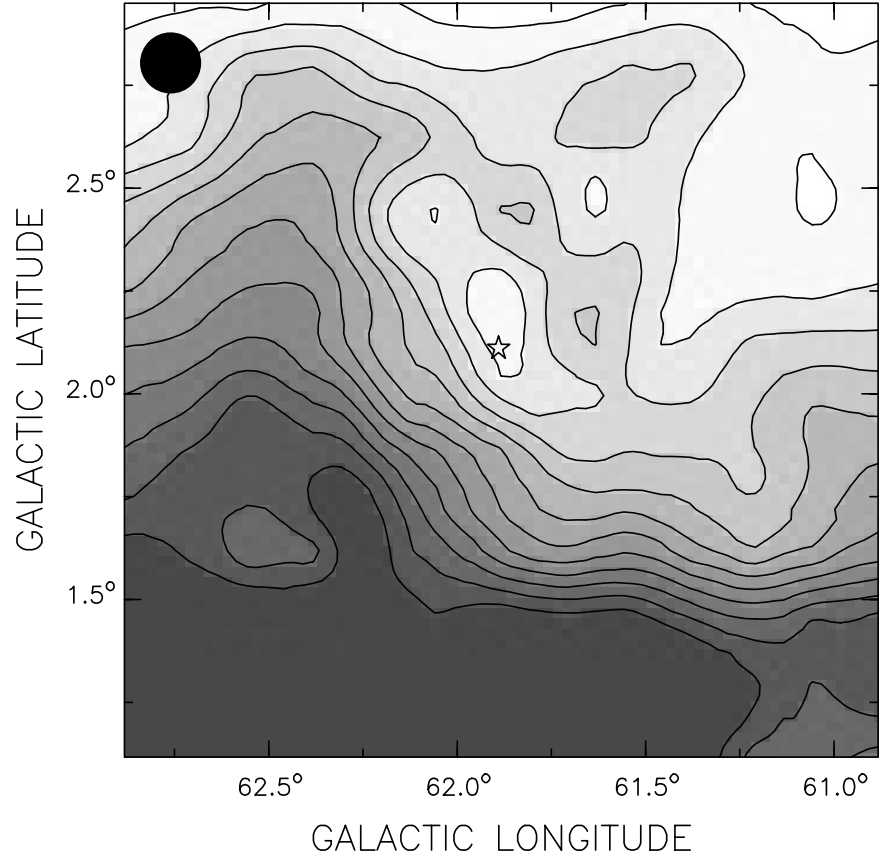

Fig. 2. WR 126 Average HI distribution in the velocity interval 20.1 to $25.2 \mathrm{~km} \mathrm{~s}^{-1}$. The star symbol indicates the optical position of WR 126. The half beam width (HPBW $\sim 9^{\prime}$ ) is shown in the upper left corner. Contour levels are at -24 to $20 \mathrm{~K}$ in steps of $4 \mathrm{~K}$.

studying large angular scale structures due to its low angular resolution $(\mathrm{HPBW}=35.5)$. From this analysis, no obvious large scale HI void that could be related to the four WR stars was found. An inspection of the available radio continuum surveys at $2695 \mathrm{MHz}$ (Fürst et al. 1990) and $4850 \mathrm{MHz}$ (Condon et al. 1994) and IRAS images (Wheelock et al. 1994) also fails to reveal any large scale feature likely to be related to Cep OB1. Neither WR 154 nor WR 155 has an associated optical ring nebula (Miller \& Chu 1993). Their published distances, obtained by different authors, are given in Table 1 .

\subsubsection{Analysis of the HI data: WR 154}

Figure 3 shows the HI distribution in the environs of WR 154 for the velocity interval -34.6 to $-24.3 \mathrm{~km} \mathrm{~s}^{-1}$.

The action of the stellar wind of WR 154 on its surrounding ISM is first noticeable at $-33.3 \mathrm{~km} \mathrm{~s}^{-1}$, where a slight distortion of the brightness temperature contours close to the stellar position is observed (stressed in Fig. 3 by a thick dash line). At $-32.0 \mathrm{~km} \mathrm{~s}^{-1}$, this distortion is larger and an HI minimum develops close to the stellar position. This HI minimum, centred at $(l, b)=\left(103^{\circ} .7,-1.3\right)$, is well defined up to $-26.8 \mathrm{~km} \mathrm{~s}^{-1}$.

Figure 4 shows the mean brightness temperature image within the velocity range -32.0 to $-26.8 \mathrm{~km} \mathrm{~s}^{-1}$. In this figure, a low HI emission region centred at $(l, b)=\left(103^{\circ} .7,-1.3\right)$ and surrounded by an HI envelope open towards lower galactic longitudes is clearly observed. The star is seen projected onto one of the high HI column density borders of the cavity. According to the Tycho-2 Catalogue, WR 154 has a proper motion of $\left(\mu_{l}, \mu_{b}\right)(\operatorname{mas})=(-3.5 \pm 3.1,-2.5 \pm 2.7)$. Due to the large errors involved, the direction of the stellar proper motion 


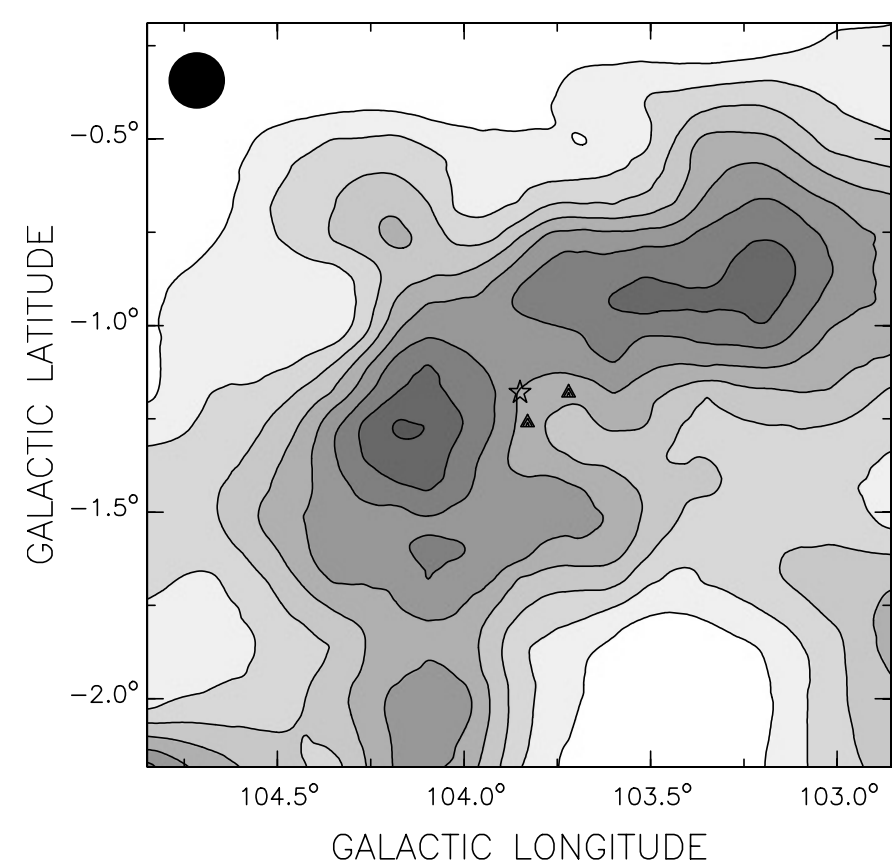

Fig. 4. WR 154 Average HI distribution in the velocity interval -32.0 to $-26.8 \mathrm{~km} \mathrm{~s}^{-1}$. The star symbol indicates the position of WR 154 and the filled triangles the position of the B stars (see text). The half beam width $\left(\right.$ HPBW $\left.\sim 9^{\prime}\right)$ is shown in the upper left corner. Contour levels are at -10 to $30 \mathrm{~K}$ in steps of $5 \mathrm{~K}$.

does not preclude a possible association between the star and the HI structure mentioned above.

Adopting for the cavity a systemic velocity of $-29 \mathrm{~km} \mathrm{~s}^{-1}$, different galactic rotation models (e.g. Ostriker \& Caldwell 1983; Blitz 1979) predict a kinematical distance of about $3.0 \pm 0.7 \mathrm{kpc}$. On the other hand, an inspection of the observed radial velocity field of the Galaxy derived by Brand \& Blitz (1993) gives for the adopted systemic velocity a distance of about $2 \pm 0.3 \mathrm{kpc}$. Based on these values, we shall adopt a distance of $2 \mathrm{kpc}$ for the HI structure. Taking into account that the distances given by van der Hucht (2001) have an error of about $50 \%$, we can consider that the adopted kinematical distance is in reasonable agreement with the catalogued ones (see Table 1). The adopted distance is also in relative good agreement with the catalogued distance of Cep OB1 (Garmany \& Stencel 1992).

The location of WR 154 and the similar distances of both the HI minimum and the WR star, makes a physical relationship between them plausible. The physical parameters of the HI structure are summarized in Table 2.

\subsubsection{Analysis of the HI data: WR 155}

In Fig. 5 a montage of HI brightness temperature images spanning the velocity range from -34.6 to $-24.3 \mathrm{~km} \mathrm{~s}^{-1}$ is shown.

At $-34.6 \mathrm{~km} \mathrm{~s}^{-1}$ WR 155 is seen projected onto a relative maximum of $\mathrm{HI}$ emission. In contrast, at $-33.3 \mathrm{~km} \mathrm{~s}^{-1}$ a noticeable relative minimum with the WR star placed inside it is clearly seen. This minimum is open towards higher galactic latitudes and remains observable till $-25.6 \mathrm{~km} \mathrm{~s}^{-1}$.
In Fig. 6 the HI brightness temperature integrated between -30.7 and $-25.6 \mathrm{~km} \mathrm{~s}^{-1}$ is shown. There, an HI cavity surrounded by regions of enhanced emission that look like an incomplete ring of neutral gas is depicted. WR 155 appears projected close to the centre of the cavity. The stellar proper motion as given in the Tycho- 2 Catalogue, $\left(\mu_{l}, \mu_{b}\right)($ mas $)=$ $(-6.1 \pm 1.6,-2.1 \pm 1.7)$, may explain the slightly eccentric position of the star with respect to the cavity symmetry centre.

It is important to note that the velocity range covered by the HI structure associated with WR 155 is similar to the one related to WR 154 (see Sect. 4.2.1). This agreement is expected since both stars are probably members of Cep OB1. As a consequence, relying on the same arguments put forward in Sect. 4.2.1, we shall adopt a kinematical distance of $2 \mathrm{kpc}$ for this HI minimum as well.

Likewise the case of WR 154, based on both the spatial distribution and the distance agreement, we conclude that the WR star and the HI minimum are very likely related to each other. Its main parameters are also given in Table 2.

It is worth mentioning that since the centroids of the HI cavities created by WR 154 and WR 155 are 1.7 apart and their mean angular radius is $\sim 0.2$, at present the individual bubbles are not interacting with each other. Owing to the similarity in the missing HI density quoted for both bubbles and their observed dimensions, their ages are likely to be comparable.

\subsubsection{Other massive stars in the field}

A search of the existing literature shows that in the vicinity of both WR 154 and WR 155 there are many catalogued early type stars. In this section, we shall attempt to identify those which might be related to the observed HI structures. Using SIMBAD, two B stars are found near WR 154 and one close to WR 155 (their positions are indicated by filled triangles in Fig. 4 and Fig. 6, respectively). A few arcminutes away from WR 155 there is also a catalogued OB star (Stock et al. 1960). Its position is marked by an open triangle in Fig. 6.

In order to check whether these stars may have contributed to the creation of the observed structures, we need to estimate the total mechanical luminosity injected by every star. Since these objects lack information about their luminosity class, distance and visual absorption, to perform the calculation mentioned above we assume that the $\mathrm{OB}$ and the three B stars are main sequence objects having a distance and reddening similar to that of the corresponding WR star.

For the B stars, an absolute magnitude is estimated from the observed $V$ magnitude and the adopted WR distance. For the OB star, using its observed $B$ magnitude and the work of Reed \& Nyman (1996) a rough absolute magnitude is obtained. In both cases, using the Schmidt-Kaler (1982) calibration an aproximate MK spectral type is derived for every star. For the B stars near WR 154 spectral types B1V and B3V are derived. A B5 V spectral type is obtained for the B star close to WR 155, while for the OB star a spectral type B1/B2 V is obtained.

Using solar abundances, typical mass loss rates $(\dot{M})$ and wind terminal velocities $\left(v_{\infty}\right)$ are computed using the formulae of Leitherer et al. (1992). The total mechanical 
luminosity $\left(L_{w}=\frac{1}{2} \dot{M} V_{\infty}\right)$ injected by these stars are about $1.3 \times 10^{33} \mathrm{erg} \mathrm{s}^{-1}(\mathrm{~B} 1 \mathrm{~V}), 5.1 \times 10^{31} \mathrm{erg} \mathrm{s}^{-1}(\mathrm{~B} 3 \mathrm{~V})$, and $1.2 \times$ $10^{30} \mathrm{erg} \mathrm{s}^{-1}(\mathrm{~B} 5 \mathrm{~V})$. All these estimates are well below the mechanical luminosity injected by WR $154\left(\sim 4.1 \times 10^{37} \mathrm{erg} \mathrm{s}^{-1}\right)$ and WR $155\left(\sim 5.4 \times 10^{37} \mathrm{erg} \mathrm{s}^{-1}\right)$. Therefore, the WR stars are by far the main sources of mechanical energy input to their local ISM.

\subsubsection{Global remarks}

Though in most of the HI bubbles detected so far the dominant morphology is an ovoidal one having a single HI minimum with the star offset from its centre, there is a small subset that shows two well-developed HI minima within a larger HI cavity. Intermediate or high angular resolution $\mathrm{HI}$ observations are required to observationally identify these dual-lobed structures. A search of the available literature indicates that the ISM local to about 25 galactic $\mathrm{O}$ and WR stars has been observed in the HI- $21 \mathrm{~cm}$ line using radiotelescopes with angular resolutions higher than $9^{\prime}$ (corresponding to the $100 \mathrm{~m}$ Effelsberg radiotelescope). Including the dual-lobed HI structure associated with WR 126, the percentage of known HI bubbles depicting this peculiarity amounts to about $15 \%$.

The values for $C_{\mathrm{ax}}$ and $C_{\mathrm{off}}$ listed in Table 2 are similar to those found for HI bubbles already known to be related to galactic O and WR stars (e.g. Arnal 1992; Cappa et al. 1996; Arnal et al. 1999; Cappa \& Herbstmeier 2000; Cichowolski et al. 2001; Cichowolski et al. 2003). It is worth reminding the reader that $C_{\mathrm{ax}}>1$ implies that the $\mathrm{HI}$ bubbles are ovoidal rather than spherical in shape, and $C_{\text {off }} \neq 0$ indicates that the star responsable for blowing the bubble is offset from its centre of symmetry. These facts can be accounted for by relaxing some of the basic assumptions adopted in the classical evolutionary models of interstellar bubbles. Indeed, the presence of density gradients in the ISM local to the star, a star having a high spatial velocity with respect to its local ISM, a non-isotropic stellar mass loss rate (e.g. Weaver et al. 1977; Marchenko 1994), and the effects of stellar and/or interstellar magnetic fields (Chevalier \& Luo 1994) can easily explain the origin of aspherical interstellar bubbles.

\section{Conclusions}

In this paper we have analyzed the HI distribution around three WR stars, namely, WR 126, WR 154 and WR 155. The results can be summarized as follows:

1. For every star, a low emissivity cavity in the HI distribution was found. These minima are interpreted as the observable $21 \mathrm{~cm}$ counterpart of the interstellar bubble created by the powerful winds of the WR stars and their progenitors.

2. These HI minima can be traced along a velocity range of $8-9 \mathrm{~km} \mathrm{~s}^{-1}$. The kinematical distances, as obtained from the systemic velocities of the HI minimum, are in good agreement with the stellar catalogued distances.

3. The cavities are ovoid structures having a major and minor axis in the range 13-27 pc and 9-10 pc, respectively. The corresponding axial ratio $C_{\mathrm{ax}}$ ranges from 1.3 to 3 .

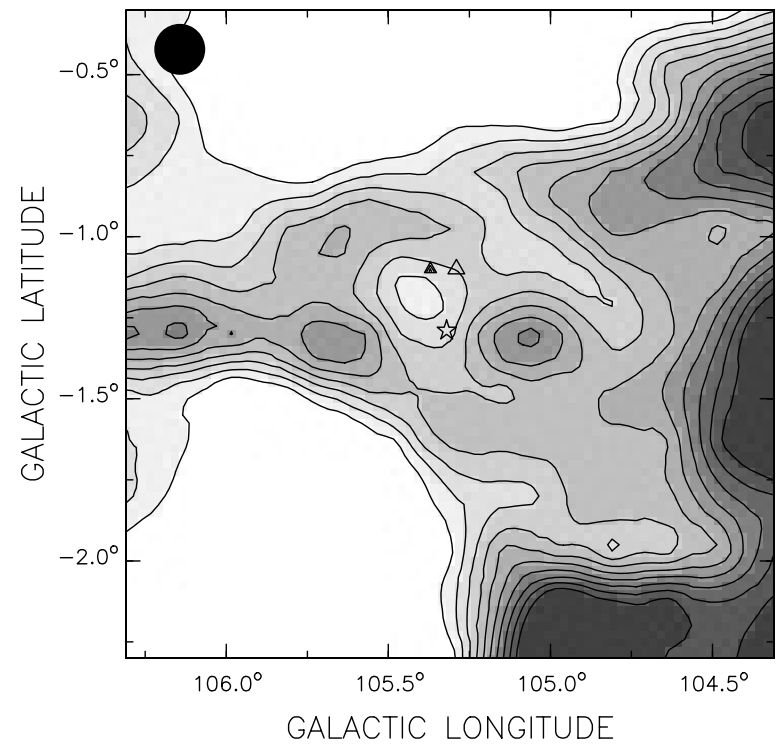

Fig. 6. WR 155 Average HI distribution in the velocity interval -30.7 to $-25.6 \mathrm{~km} \mathrm{~s}^{-1}$. The star symbol indicates the position of WR 155 . The filled and open triangles indicate the position of the B and OB star, respectively (see text). The half beam width $\left(\mathrm{HPBW} \sim 9^{\prime}\right)$ is shown in the upper left corner. Contour levels are at -4 to $14 \mathrm{~K}$ in steps of $2 \mathrm{~K}$.

4. In all the cases, the WR star is eccentric with respect to the symmetry centre of the observed cavity. The offset parameter $C_{\text {off }}$ varies between 0.5 and 0.8 .

5. The HI cavity observed around WR 126 may be another case of a dual-lobed like feature.

Acknowledgements. E.M.A. thanks the staff of the Max-PlanckInstitüt für Radioastronomie for their hospitality during his stay. S.C. wishes to thank the Director of the Instituto de Astronomía y Física del Espacio (IAFE), Dr. Marta Rovira, for allowing her the use of the IAFE facilities. It is also a pleasure to thank Dr. S. Pineault for his comments on an early version of this manuscript. We would like to thank the referee for her/his constructive comments and suggestions that help us to clarify some points. This work was partially financed by Consejo Nacional de Investigaciones Científicas y Técnicas (CONICET) of Argentina, under project PIP 4252/96.

\section{References}

Arnal, E. M. 1992, A\&A, 254, 305

Arnal, E. M. 2001, AJ, 121, 413

Arnal, E. M., \& Cappa, C. E. 1996, MNRAS, 279, 788

Arnal, E. M., \& Roger, R. S. 1997, MNRAS, 285, 253

Arnal, E. M., Cappa, C. E., Rizzo, J. R., \& Cichowolski S. 1999, AJ, 118,1798

Avedisova, V. 1972, Soviet Astron., 15, 708

Blitz, L. 1979, ApJ, 231, L118

Brand, J., \& Blitz, L. 1993, A\&A, 275, 67

Cappa, C. E., \& Herbstmeier, U. 2000, AJ, 120, 1963

Cappa, C. E., Niemela, V. S., Herbstmeier, U., \& Koribalski, B. 1996, A\&A, 312, 283

Castor, J. I., McCray, R., \& Weaver, R. 1975, ApJ, 200, L107

Chevalier, R. A., \& Luo, D. 1994, ApJ, 421, 225

Cichowolski, S., Pineault, S., Arnal, E. M., et al. 2001, AJ, 122, 1938 
Cichowolski, S., Arnal, E. M., Cappa, C. E., Pineault, S., \& St-Louis, N. 2003, MNRAS, 343, 47

Condon, J. J., Broderick, J. J., \& Seielstad, G. A. 1989, AJ, 97, 1064

Condon, J. J., Broderick, J. J., Seielstad, G. A., Douglas, K., \& Gregory, P. C. 1994, AJ, 107, 1829

Conti, P. S., \& Vacca, W. D. 1990, AJ, 100, 431

Fürst, E., Reich, W., Reich, P., \& Reif, K. 1990, A\&AS, 85, 691

García-Segura, G., \& Mac Low, M. 1995a, ApJ, 455, 145

García-Segura, G., \& Mac Low, M. 1995b, ApJ, 455, 160

Garmany, C. D., \& Stencel, R. E. 1992, A\&AS, 94, 211

Harten, R. H., Felli, M., \& Tofani, G. 1978, A\&A, 70, 205

van der Hucht, K. A. 2001, New Astron. Rev., 45, 135

Kalberla, P. M. W., Mebold, U., \& Reich, W. 1980, A\&A, 82, 275

Leitherer, C., Robert, C., \& Drissen, L. 1992, ApJ, 401, 596

Lundström, I., \& Stenholm, B. 1984, A\&AS, 58, 163

Meynet, G., \& Maeder, A. 2003, A\&A, 404, 975

Marchenko, S. V. 1994, Ap\&SS, 221, 169

Miller, G. J., \& Chu, Y. H. 1993, ApJS, 85, 137

Ostriker, J. P., \& Caldwell, J. A. R. 1983, in Kinematics, Dynamics and Structure of the Milky Way, Astrophys. Space Sci. Library, 100, 249
Pineault, S. 1998, AJ, 115, 2483

Reed, B. C., \& Nyman, M. A. 1996, PASP, 108395

Ruprecht, J., Balázs, B., \& White, R. E. 1981, Catalogue of Star Clusters and Associations, Suppl. 1, part B2, 419

Schmidt-Kaler, Th. 1982, Landolt-Börnstein, New Series, Group 6, vol. 2b, ed. K. Schaiffers, \& H. H. Voigt (Berlin: Springer), Chap. 4.1

Smith, L. F., Shara, M. M., \& Moffat, A. F. J. 1990, ApJ, 358, 229

Stock, J., Nassau, J. J., \& Stephenson, C. B. 1960, Luminous Stars in the Northen Milky Way, vol. 2 (Hamburg-Bergedorf: Hamburger Sternwarte and Warner Swasey Obs.)

Weaver, H., \& Williams, D. R. H. 1973, A\&AS, 8, 1

Weaver, R., McCray, R., Castor, J. I., Shapiro, P., \& Moore, R. 1977, ApJ, 218, 377

Wheelock, S. L., Gautier, T. N., Chillemi, J., et al. 1994, IRAS Sky Survey Atlas Explanatory Suppl. (JPL Publ. 94-11) (Pasadena:JPL)

White, R. L., \& Becker, R. H. 1992, ApJS, 79, 331

Williams, D. R. W. 1973, A\&AS, 8, 505 
S. Cichowolski and E. M. Arnal: An Effelsberg HI study of the ISM around WR 126, WR 154 and WR 155, Online Material p 1

\section{Online Material}


S. Cichowolski and E. M. Arnal: An Effelsberg HI study of the ISM around WR 126, WR 154 and WR 155, Online Material p 2
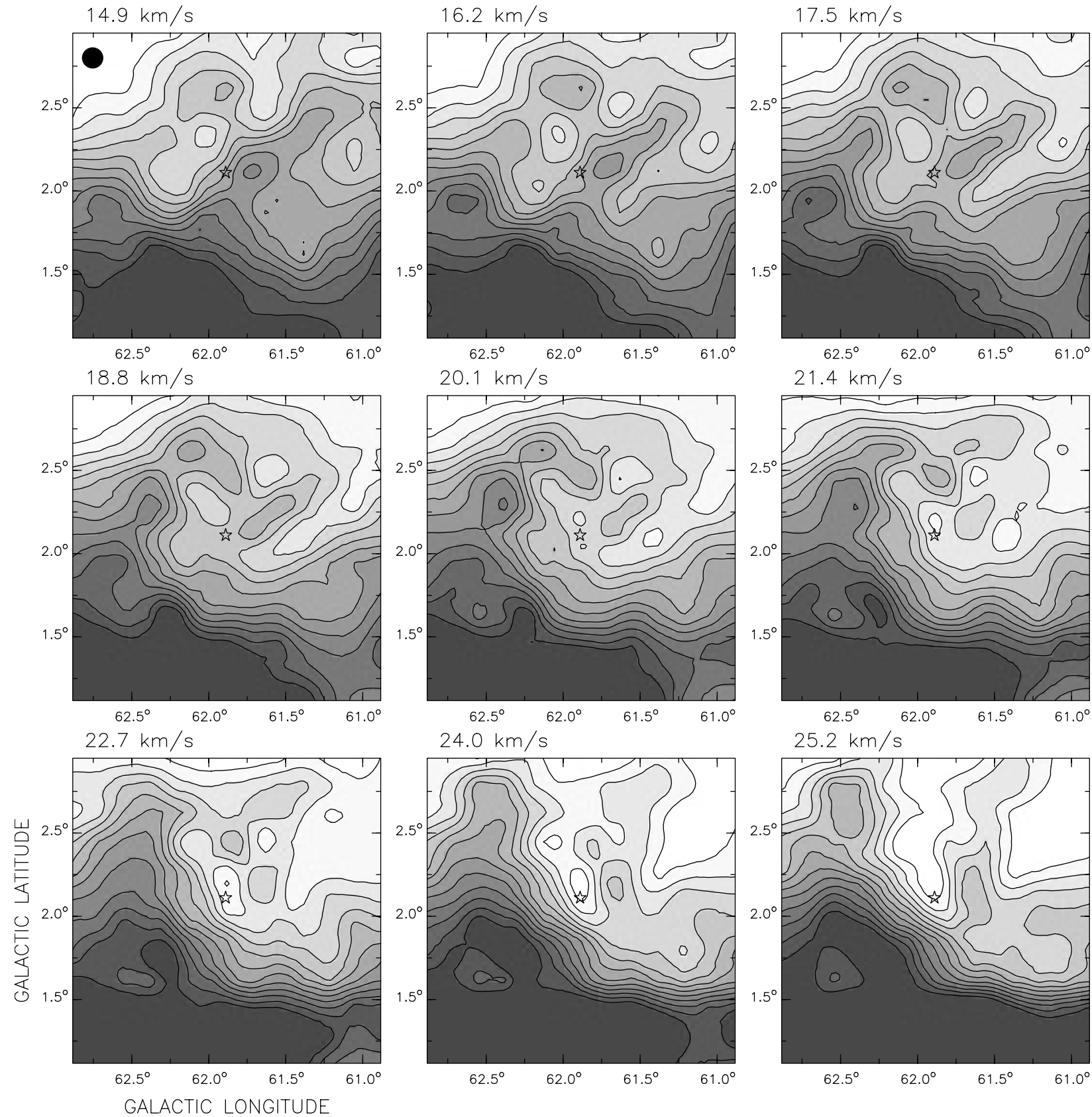

Fig. 1. WR 126 Effelsberg grey-scale images showing the HI distribution in the velocity range 14.9 to $25.2 \mathrm{~km} \mathrm{~s}^{-1}$. The half beam width $\left(H P B W \sim 9^{\prime}\right)$ is shown in the upper left corner of the first image. The stellar position is indicated by a star symbol. The central velocity of every image is given in the upper left corner. Contour levels are at -24 to $20 \mathrm{~K}$ in steps of $4 \mathrm{~K}$. For all images, darker shadings means higher brightness temperature. 
S. Cichowolski and E. M. Arnal: An Effelsberg HI study of the ISM around WR 126, WR 154 and WR 155, Online Material p 3
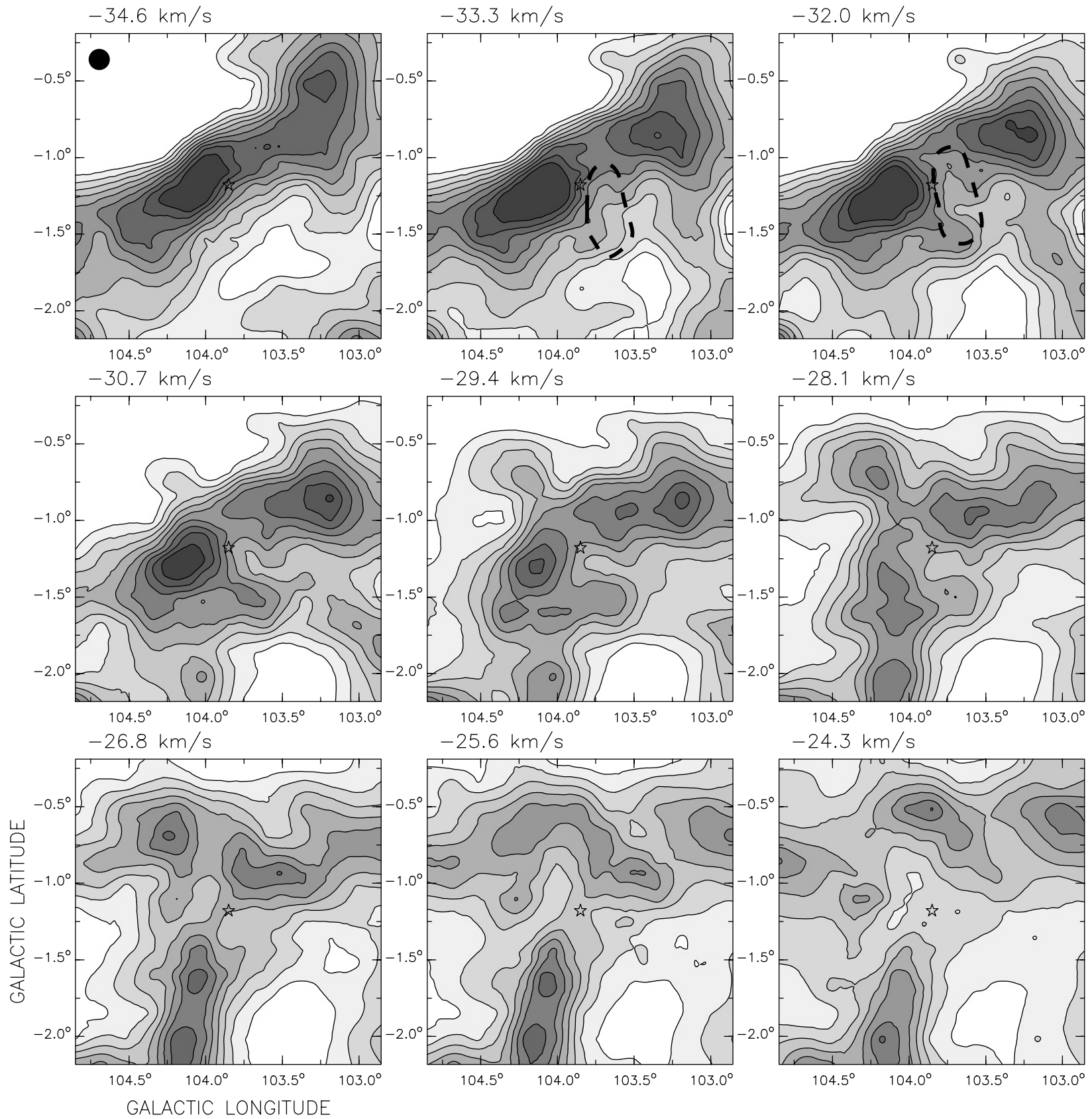

Fig. 3. WR 154 Effelsberg grey-scale images showing the HI distribution in the velocity range -34.6 to $-24.3 \mathrm{~km} \mathrm{~s}^{-1}$. The half beam width (HPBW $\sim 9^{\prime}$ ) is shown in the upper left corner of the first image. The stellar position is indicated by a star symbol. The central velocity of every map is specified in the upper left corner. Contour levels are at -10 to $30 \mathrm{~K}$ in steps of $5 \mathrm{~K}$. 
S. Cichowolski and E. M. Arnal: An Effelsberg HI study of the ISM around WR 126, WR 154 and WR 155, Online Material p 4
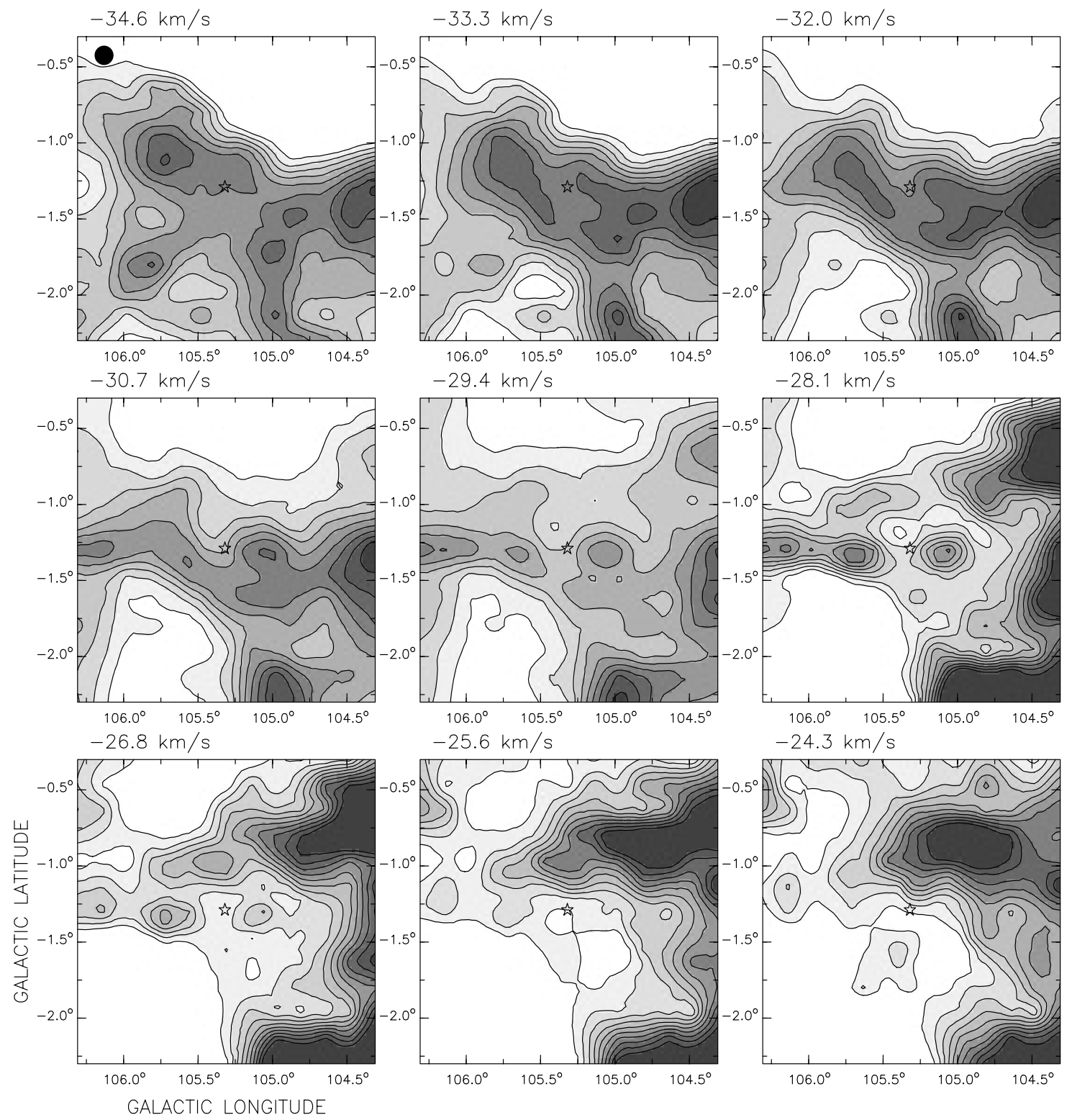

Fig. 5. WR 155 Effelsberg grey-scale images showing the HI distribution in the velocity range -34.6 to $-24.3 \mathrm{~km} \mathrm{~s}^{-1}$. The half beam width (HPBW $\sim 9^{\prime}$ ) is shown in the upper left corner of the first image. The stellar position is indicated by a star symbol. The central velocity of every image is specified in the upper left corner. Contour levels are at -10 to $30 \mathrm{~K}$ in steps of $5 \mathrm{~K}$ for the velocity range -34.6 to $-29.4 \mathrm{~km} \mathrm{~s}{ }^{-1}$ and at -4 to $14 \mathrm{~K}$ in steps of $2 \mathrm{~K}$ for the rest. 